\title{
Additional compact formulas for vibrational dynamic dipole polarizabilities and hyperpolarizabilities
}

\author{
David M. Bishop \\ Department of Chemistry, University of Ottawa, Ottawa K1N 6N5, Canada \\ Josep M. Luis ${ }^{\text {a) }}$ and Bernard Kirtman \\ Department of Chemistry, University of California, Santa Barbara, California 93106
}

(Received 29 December 1997; accepted 20 March 1998)

\begin{abstract}
Compact expressions, complete through second order in electrical and/or mechanical anharmonicity, are given for the dynamic dipole vibrational polarizability and dynamic first and second vibrational hyperpolarizabilities. Certain contributions not previously formulated are now included. (C) 1998 American Institute of Physics. [S0021-9606(98)02324-1]
\end{abstract}

The past decade has seen an increasing number of calculations of vibrational polarizabilities and hyperpolarizabilities. At this time, some hundred-odd papers have touched on this subject, and it has recently been reviewed in detail by one of us. ${ }^{1}$ For some systems and processes these vibrational properties are even more important than the corresponding electronic ones. Together both contributions govern nonlinear optical (NLO) behavior. A large number of the vibrational calculations have been based on one or both of two approximations: (a) the vibrations are assumed to be harmonic, and the electronic properties are assumed to be linear in the normal coordinates-this is the double-harmonicoscillator approximation; ${ }^{2}$ and (b) the optical frequencies are taken to be infinite ${ }^{2}$ - this is the enhanced, or infinite frequency approximation. ${ }^{3,4}$ These approximations have been analyzed by Bishop and Dalskov. ${ }^{5}$ The only rigorous treatment of the dynamic vibrational (hyper)polarizabilities is that given by Bishop and Kirtman ${ }^{6}$ in the early nineties. The purpose here is to extend their general formulas so that they are complete through second order of perturbation theory in mechanical and/or electrical anharmonicity. All vibrational effects are included except for zero-point vibrational averaging of the electronic (hyper)polarizability.

We write the vibrational polarizability $\left(\alpha^{v}\right)$ and the first and second vibrational hyperpolarizability $\left(\beta^{v}\right.$ and $\left.\gamma^{v}\right)$ as a sum of terms derived from the sum-over-states expressions for the total (hyper)polarizability, which are given in terms of vibronic energies and dipole moment matrix elements over the vibronic wave functions, ${ }^{7}$

$$
\begin{aligned}
& \alpha^{v}\left(-\omega_{\sigma} ; \omega_{1}\right)=\left[\mu^{2}\right], \\
& \beta^{v}\left(-\omega_{\sigma} ; \omega_{1}, \omega_{2}\right)=[\mu \alpha]+\left[\mu^{3}\right], \\
& \gamma^{v}\left(-\omega_{\sigma} ; \omega_{1}, \omega_{2}, \omega_{3}\right)=\left[\alpha^{2}\right]+[\mu \beta]+\left[\mu^{2} \alpha\right]+\left[\mu^{4}\right] .
\end{aligned}
$$

Here, the optical frequencies $\left(\omega_{1}, \omega_{2}, \omega_{3}\right)$ define a particular NLO process and $\omega_{\sigma}=\Sigma_{i} \omega_{i}$. The square-bracket terms ${ }^{6}$ are sums over vibrational states of a quotient in which the nu-

\footnotetext{
${ }^{a)}$ Permanent address: Institute of Computational Chemistry and Department of Chemistry, University of Girona, 17017 Girona, Catalonia, Spain.
}

merator introduces vibrational transition matrix elements of the electronic properties $(\mu, \alpha, \beta)$ and the denominator involves the vibrational and optical frequencies. For example,

$$
\begin{aligned}
{[\mu \alpha]=} & \frac{1}{2 \hbar} \sum P_{\alpha \beta \gamma} \sum_{k}^{\prime}\left(\mu_{\alpha}\right)_{0 k}\left(\alpha_{\beta \gamma}\right)_{k 0}\left[\left(\omega_{k}+\omega_{\sigma}\right)^{-1}\right. \\
& \left.+\left(\omega_{k}-\omega_{\sigma}\right)^{-1}\right]
\end{aligned}
$$

where $\left(\mu_{\alpha}\right)_{0 k}=\left\langle 0\left|\mu_{\alpha}\right| k\right\rangle ;|k\rangle$ is the $k$ th vibrational wave function with energy $\hbar \omega_{k} ; \mu_{\alpha}$ is the $\alpha$ component of the dipole moment $(\alpha, \beta, \gamma=$ Cartesian coordinates $x, y, z)$; $\sum P_{\alpha \beta \gamma}$ indicates a sum over the simultaneous permutations of frequencies and subscripts $\left(-\omega_{\sigma}, \alpha\right),\left(\omega_{1}, \beta\right)$, and $\left(\omega_{2}, \gamma\right)$ (an alternative equivalent notation is $\left.\Sigma P_{-\sigma, 1,2}\right)$; and the prime on the second summation denotes exclusion of the vibrational ground state.

The electronic properties are, next, expanded in the normal coordinates $\left(Q_{a}, Q_{b}, \ldots\right)$, e.g.,

$$
\begin{aligned}
\mu_{\alpha} \cong & \mu_{\alpha}^{0}+\sum_{a}\left(\frac{\partial \mu}{\partial Q_{a}}\right) Q_{a}+\frac{1}{2} \sum_{a, b}\left(\frac{\partial^{2} \mu}{\partial Q_{a} \partial Q_{b}}\right) Q_{a} Q_{b} \\
& +\frac{1}{6} \sum_{a, b, c}\left(\frac{\partial^{3} \mu}{\partial Q_{a} \partial Q_{b} \partial Q_{c}}\right) Q_{a} Q_{b} Q_{c} .
\end{aligned}
$$

Here the quadratic term is considered to be first order in electrical anharmonicity and the cubic term is considered to be second order. Thus, it is sufficient for our purposes to truncate the expansion as above after the third derivatives. The vibrational potential, $V$, is expanded in a similar manner,

$$
\begin{aligned}
V \cong & V^{0}+\frac{1}{2} \sum_{a} \omega_{a}^{2} Q_{a}^{2}+\frac{1}{6} \sum_{a, b, c} F_{a b c} Q_{a} Q_{b} Q_{c} \\
& +\frac{1}{24} \sum_{a, b, c, d} F_{a b c d} Q_{a} Q_{b} Q_{c} Q_{d},
\end{aligned}
$$


TABLE I. Formulas for the contributions to dynamic vibrational polarizabilities and hyperpolarizabilities. ${ }^{\mathrm{a}}$

\begin{tabular}{|c|c|}
\hline Term & Formula \\
\hline$\left[\mu^{2}\right]^{0,0}$ & $\frac{1}{2} \Sigma P_{\alpha \beta} \Sigma_{a}\left(\partial \mu_{\alpha} / \partial Q_{a}\right)\left(\partial \mu_{\beta} / \partial Q_{a}\right) \lambda_{a}^{ \pm \sigma}$ \\
\hline$\left[\mu^{2}\right]^{2,0}$ & $\begin{array}{l}(\hbar / 8) \sum P_{\alpha \beta} \Sigma_{a, b}\left[\left(\partial^{2} \mu_{\alpha} / \partial Q_{a} \partial Q_{b}\right)\left(\partial^{2} \mu_{\beta} / \partial Q_{a} \partial Q_{b}\right)\left(\omega_{a}^{-1}\right.\right. \\
\left.\quad+\omega_{b}^{-1}\right) \lambda_{a b}^{ \pm \sigma}+\left(\partial^{3} \mu_{\alpha} / \partial Q_{a}^{2} \partial Q_{b}\right)\left(\partial \mu_{\beta} / \partial Q_{b}\right) \omega_{a}^{-1} \lambda_{b}^{ \pm \sigma} \\
\left.\quad+\left(\partial \mu_{\alpha} / \partial Q_{b}\right)\left(\partial^{3} \mu_{\beta} / \partial Q_{a}^{2} \partial Q_{b}\right) \omega_{a}^{-1} \lambda_{b}^{ \pm \sigma}\right]\end{array}$ \\
\hline$\left[\mu^{2}\right]^{1,1}$ & $\begin{aligned}- & (\hbar / 4) \Sigma P_{\alpha \beta} \Sigma_{a, b, c}\left[F_{a b c}\left(\partial^{2} \mu_{\alpha} / \partial Q_{a} \partial Q_{b}\right)\left(\partial \mu_{\beta} / \partial Q_{c}\right)\right. \\
& \times\left(\omega_{a}^{-1}+\omega_{b}^{-1}\right) \lambda_{a b}^{+\sigma} \lambda_{c}^{ \pm \sigma}+F_{b c c}\left(\partial^{2} \mu_{\alpha} / \partial Q_{a} \partial Q_{b}\right) \\
& \left.\times\left(\partial \mu_{\beta} / \partial Q_{a}\right) \omega_{b}^{-2} \omega_{c}^{-1} \lambda_{a}^{ \pm \sigma}\right]\end{aligned}$ \\
\hline$\left[\mu^{2}\right]^{0,2}$ & $\begin{aligned}- & (\hbar / 8) \Sigma P_{\alpha \beta} \Sigma_{a, b, c} \omega_{a}^{-1}\left[F_{a a b c}\left(\partial \mu_{\alpha} / \partial Q_{b}\right)\right. \\
& \times\left(\partial \mu_{\beta} / \partial Q_{c}\right) \lambda_{b}^{ \pm \sigma} \lambda_{c}^{ \pm \sigma}-\Sigma_{d}\left\{F_{a a b} F_{b c d}\left(\partial \mu_{\alpha} / \partial Q_{c}\right)\right. \\
& \times\left(\partial \mu_{\beta} / \partial Q_{d}\right) \omega_{b}^{-2} \lambda_{c}^{ \pm \sigma} \lambda_{d}^{ \pm \sigma}+2 F_{a b c} F_{a b d}\left(\partial \mu_{\alpha} / \partial Q_{c}\right) \\
& \left.\left.\times\left(\partial \mu_{\beta} / \partial Q_{d}\right) \lambda_{a b}^{ \pm \sigma} \lambda_{c}^{ \pm} \lambda_{d}^{ \pm \sigma}\right\}\right]\end{aligned}$ \\
\hline$\left[\mu^{3}\right]^{1,0}$ & $\begin{array}{l}\frac{1}{2} \sum_{\alpha \beta \gamma} P_{a, b}\left(\partial \mu_{\alpha} / \partial Q_{a}\right)\left(\partial^{2} \mu_{\beta} / \partial Q_{a} \partial Q_{b}\right) \\
\quad \times\left(\partial \mu_{\gamma} / \partial Q_{b}\right) \lambda_{a}^{ \pm \sigma} \lambda_{b}^{ \pm 2}\end{array}$ \\
\hline$\left[\mu^{3}\right]^{0,1}$ & $\begin{aligned}- & \frac{1}{6} \Sigma P_{\alpha \beta \gamma} \Sigma_{a, b, c} F_{a b c}\left(\partial \mu_{\alpha} / \partial Q_{a}\right)\left(\partial \mu_{\beta} / \partial Q_{b}\right) \\
& \times\left(\partial \mu_{\gamma} / \partial Q_{c}\right) \lambda_{a}^{ \pm \sigma} \lambda_{b}^{ \pm 1} \lambda_{c}^{ \pm 2}\end{aligned}$ \\
\hline$\left[\mu^{2} \alpha\right]^{1,0}$ & $\begin{array}{l}\frac{1}{4} \Sigma P_{\alpha \beta \gamma \delta} \Sigma_{a, b}\left[\left(\partial \mu_{\alpha} / \partial Q_{a}\right)\left(\partial^{2} \alpha_{\beta \gamma} / \partial Q_{a} \partial Q_{b}\right)\right. \\
\quad \times\left(\partial \mu_{\delta} / \partial Q_{b}\right) \lambda_{a}^{ \pm \sigma} \lambda_{b}^{ \pm 3}+2\left(\partial \mu_{\alpha} / \partial Q_{a}\right)\left(\partial^{2} \mu_{\beta} / \partial Q_{a} \partial Q_{b}\right) \\
\left.\quad \times\left(\partial \alpha_{\gamma \delta} / \partial Q_{b}\right) \lambda_{a}^{ \pm \sigma} \lambda_{b}^{ \pm 23}\right]\end{array}$ \\
\hline$\left[\mu^{2} \alpha\right]^{0,1}$ & $\begin{array}{l}-\frac{1}{4} \sum P_{\alpha \beta \gamma \delta} \Sigma_{a, b, c} F_{a b c}\left(\partial \mu_{\alpha} / \partial Q_{a}\right)\left(\partial \mu_{\beta} / \partial Q_{b}\right) \\
\quad \times\left(\partial \alpha_{\gamma \delta} / \partial Q_{c}\right) \lambda_{a}^{ \pm \sigma} \lambda_{b}^{ \pm 1} \lambda_{c}^{ \pm 23}\end{array}$ \\
\hline$\left[\mu^{4}\right]^{2,0}$ & $\begin{aligned} \frac{1}{6} & \sum P_{\alpha \beta \gamma \delta} \Sigma_{a, b, c}\left[3\left(\partial \mu_{\alpha} / \partial Q_{a}\right)\left(\partial^{2} \mu_{\beta} / \partial Q_{a} \partial Q_{b}\right)\right. \\
& \times\left(\partial^{2} \mu_{\gamma} / \partial Q_{b} \partial Q_{c}\right)\left(\partial \mu_{\delta} / \partial Q_{c}\right) \lambda_{a}^{ \pm \sigma} \lambda_{b}^{ \pm 23} \lambda_{c}^{ \pm 3} \\
& +\left(\partial^{3} \mu_{\alpha} / \partial Q_{a} \partial Q_{b} \partial Q_{c}\right)\left(\partial \mu_{\beta} / \partial Q_{a}\right)\left(\partial \mu_{\gamma} / \partial Q_{b}\right) \\
& \left.\times\left(\partial \mu_{\delta} / \partial Q_{c}\right) \lambda_{a}^{ \pm 1} \lambda_{b}^{ \pm 2} \lambda_{c}^{ \pm 3}\right]\end{aligned}$ \\
\hline$\left[\mu^{4}\right]^{1,1}$ & $\begin{aligned}- & \frac{1}{2} \sum P_{\alpha \beta \gamma \delta} \Sigma_{a, b, c, d} F_{a b c}\left(\partial \mu_{\alpha} / \partial Q_{a}\right)\left(\partial \mu_{\beta} / \partial Q_{b}\right) \\
& \times\left(\partial^{2} \mu_{\gamma} / \partial Q_{c} \partial Q_{d}\right)\left(\partial \mu_{\delta} / \partial Q_{d}\right) \lambda_{a}^{ \pm \sigma} \lambda_{b}^{ \pm 1} \lambda_{c}^{ \pm 23} \lambda_{d}^{ \pm 3}\end{aligned}$ \\
\hline$\left[\mu^{4}\right]^{0,2}$ & $\begin{aligned}- & \frac{1}{24} \sum P_{\alpha \beta \gamma \delta} \Sigma_{a, b, c, d}\left[F_{a b c d}\left(\partial \mu_{\alpha} / \partial Q_{a}\right)\left(\partial \mu_{\beta} / \partial Q_{b}\right)\right. \\
& \times\left(\partial \mu_{\gamma} / \partial Q_{c}\right)\left(\partial \mu_{\delta} / \partial Q_{d}\right) \lambda_{a}^{ \pm \sigma} \lambda_{b}^{ \pm 1} \lambda_{c}^{ \pm 2} \lambda_{d}^{ \pm 3} \\
& -3 \Sigma_{e} F_{a b c} F_{c d e}\left(\partial \mu_{\alpha} / \partial Q_{a}\right)\left(\partial \mu_{\beta} / \partial Q_{b}\right)\left(\partial \mu_{\gamma} / \partial Q_{d}\right) \\
& \left.\times\left(\partial \mu_{\delta} / \partial Q_{e}\right) \lambda_{a}^{ \pm \sigma} \lambda_{b}^{ \pm 1} \lambda_{c}^{ \pm 23} \lambda_{d}^{ \pm 2} \lambda_{e}^{ \pm 3}\right]\end{aligned}$ \\
\hline
\end{tabular}

${ }^{a}$ Abbreviations:

(i) For the $\left[\mu^{2}\right]$ terms, $\omega_{\sigma}=\omega_{1}$; for the $\left[\mu^{3}\right]$ and $\left[\mu^{2} \alpha\right]$ terms, $\omega_{\sigma}=\omega_{1}+\omega_{2}$; for the $\left[\mu^{4}\right]$ terms, $\omega_{\sigma}$ $=\omega_{1}+\omega_{2}+\omega_{3}$.

(ii) $\Sigma P_{\alpha \beta}$ indicates the sum-over-terms generated by the permutation of the pairs of optical frequencies and indices: $\left(-\omega_{\sigma}, \alpha\right)$ and $\left(\omega_{1}, \beta\right) ; \sum P_{\alpha \beta \gamma}$ indicates the permutations of $\left(-\omega_{\sigma}, \alpha\right),\left(\omega_{1}, \beta\right)$, and $\left(\omega_{2}, \gamma\right)$; $\sum P_{\alpha \beta \gamma \delta}$ indicates the permutations of $\left(-\omega_{\sigma}, \alpha\right),\left(\omega_{1}, \beta\right),\left(\omega_{2}, \gamma\right)$, and $\left(\omega_{3}, \delta\right)$.

(iii) $\lambda_{x}^{ \pm i}=\left(\omega_{x}^{2}-\omega_{i}^{2}\right)^{-1} ; \lambda_{x y}^{ \pm i}=\left[\left(\omega_{x}+\omega_{y}\right)^{2}-\omega_{i}^{2}\right]^{-1} ; \lambda_{x}^{ \pm i j}=\left[\omega_{x}^{2}-\left(\omega_{i}+\omega_{j}\right)^{2}\right]^{-1}$.

but, in this case, the cubic term is taken to be first order in mechanical anharmonicity and the series is terminated after the (second-order) term involving the quartic force constants, $F_{a b c d}$, which are fourth derivatives of $V$. Treating the electrical and mechanical anharmonicity by double perturbation theory and substituting into Eqs. (1)-(3) leads to

$$
\begin{aligned}
& \alpha^{v}\left(-\omega_{\sigma} ; \omega_{1}\right)=\left[\mu^{2}\right]^{0}+\left[\mu^{2}\right]^{\mathrm{II}} \\
& \beta^{v}\left(-\omega_{\sigma} ; \omega_{1}, \omega_{2}\right)=[\mu \alpha]^{0}+\left[\mu^{3}\right]^{\mathrm{I}}+[\mu \alpha]^{\mathrm{II}} \\
& \begin{aligned}
\gamma^{v}\left(-\omega_{\sigma} ; \omega_{1}, \omega_{2}, \omega_{3}\right)= & {\left[\alpha^{2}\right]^{0}+[\mu \beta]^{0}+\left[\mu^{2} \alpha\right]^{\mathrm{I}}+\left[\alpha^{2}\right]^{\mathrm{II}} } \\
& +[\mu \beta]^{\mathrm{II}}+\left[\mu^{4}\right]^{\mathrm{II}}
\end{aligned}
\end{aligned}
$$

where we have used the abbreviations []$^{0}=[]^{0,0},[]^{\mathrm{I}}$ $=[]^{1,0}+[]^{0,1}$, and []$^{\mathrm{II}}=[]^{2,0}+[]^{1,1}+[]^{0,2}$. The notation []$^{n, m}$ indicates the order of electrical anharmonicity $(n)$ and the order of mechanical anharmonicity $(m)$.

As stated in our earlier work, ${ }^{6}$ we previously neglected the cubic term in Eq. (5) and all of the []$^{0,2}$ contributions in []$^{\mathrm{II}}$. We now include these and, in order that there be a single source of information, we give the complete formulas in Tables I and II.

With the exception of that part of the $\left[\mu^{4}\right]^{0,2}$ term which contains two cubic force constants $\left(F_{a b c}\right.$ and $\left.F_{c d e}\right)$, our results were achieved by arduous algebraic manipulation. The " $F^{2}$," part of $\left[\mu^{4}\right]^{0,2}$ was found by using a sequential pattern, as discussed in Ref. 1, together with the known static 
TABLE II. Conversion of formulas for $\left[\mu^{2}\right]$ to those for $[\mu \alpha],\left[\alpha^{2}\right]$, and $[\mu \beta]$.

\begin{tabular}{|c|c|c|c|c|}
\hline $\begin{array}{c}\text { Initial } \\
\text { term }\end{array}$ & Multiply by & $\begin{array}{l}\text { Permutation sum } \\
\text { change }\end{array}$ & $\begin{array}{l}\text { Electronic property } \\
\text { change } \\
\text { and frequency change }\end{array}$ & $\begin{array}{l}\text { Final } \\
\text { term }\end{array}$ \\
\hline$\left[\mu^{2}\right]^{0,0}$ & 1 & $P_{\alpha \beta} \rightarrow P_{\alpha \beta \gamma}$ & $\mu_{\beta} \rightarrow \alpha_{\beta \gamma}$ & {$[\mu \alpha]^{0,0}$} \\
\hline$\left[\mu^{2}\right]^{0,0}$ & $\frac{1}{4}$ & $P_{\alpha \beta} \rightarrow P_{\alpha \beta \gamma \delta}$ & $\begin{array}{l}\mu_{\alpha} \rightarrow \alpha_{\alpha \beta}, \mu_{\beta} \rightarrow \alpha_{\gamma \delta} \\
\quad \lambda_{a}^{ \pm \sigma} \rightarrow \lambda_{a}^{ \pm 23}\end{array}$ & {$\left[\alpha^{2}\right]^{0,0}$} \\
\hline$\left[\mu^{2}\right]^{0,0}$ & $\frac{1}{3}$ & $P_{\alpha \beta} \rightarrow P_{\alpha \beta \gamma \delta}$ & $\mu_{\beta} \rightarrow \beta_{\beta \gamma \delta}$ & {$[\mu \beta]^{0,0}$} \\
\hline$\left[\mu^{2}\right]^{2,0}$ & 1 & $P_{\alpha \beta} \rightarrow P_{\alpha \beta \gamma}$ & $\mu_{\beta} \rightarrow \alpha_{\beta \gamma}$ & {$[\mu \alpha]^{2,0}$} \\
\hline$\left[\mu^{2}\right]^{2,0}$ & $\frac{1}{4}$ & $P_{\alpha \beta} \rightarrow P_{\alpha \beta \gamma \delta}$ & $\begin{array}{c}\mu_{\alpha} \rightarrow \alpha_{\alpha \beta}, \mu_{\beta} \rightarrow \alpha_{\gamma \delta} \\
\lambda_{a b}^{ \pm \sigma} \rightarrow \lambda_{a b}^{ \pm 23}, \lambda_{b}^{ \pm \sigma} \rightarrow \lambda_{b}^{ \pm 23}\end{array}$ & {$\left[\alpha^{2}\right]^{2,0}$} \\
\hline$\left[\mu^{2}\right]^{2,0}$ & $\frac{1}{3}$ & $P_{\alpha \beta} \rightarrow P_{\alpha \beta \gamma \delta}$ & $\mu_{\beta} \rightarrow \beta_{\beta \gamma \delta}$ & {$[\mu \beta]^{2,0}$} \\
\hline$\left[\mu^{2}\right]^{1,1}$ & $\frac{1}{2}$ & $P_{\alpha \beta} \rightarrow P_{\alpha \beta \gamma}$ & $\begin{array}{l}\text { Each term is replaced by two: } \\
\text { in the first } \mu_{\beta} \rightarrow \alpha_{\beta \gamma} \text { and in the } \\
\text { second } \mu_{\alpha} \rightarrow \alpha_{\beta \gamma} \text { and } \mu_{\beta} \rightarrow \mu_{\alpha}\end{array}$ & {$[\mu \alpha]^{1,1}$} \\
\hline$\left[\mu^{2}\right]^{1,1}$ & $\frac{1}{4}$ & $P_{\alpha \beta} \rightarrow P_{\alpha \beta \gamma \delta}$ & $\begin{array}{c}\mu_{\alpha} \rightarrow \alpha_{\alpha \beta}, \mu_{\beta} \rightarrow \alpha_{\gamma \delta} \\
\lambda_{a b}^{ \pm \sigma} \rightarrow \lambda_{a b}^{ \pm 23}, \lambda_{a}^{ \pm \sigma} \rightarrow \lambda_{a}^{ \pm 23}, \lambda_{c}^{ \pm \sigma} \rightarrow \lambda_{c}^{ \pm 23}\end{array}$ & {$\left[\alpha^{2}\right]^{1,1}$} \\
\hline$\left[\mu^{2}\right]^{1,1}$ & $\frac{1}{6}$ & $P_{\alpha \beta} \rightarrow P_{\alpha \beta \gamma \delta}$ & $\begin{array}{c}\text { Each term is replaced by two: in the } \\
\text { first } \mu_{\beta} \rightarrow \beta_{\beta \gamma \delta} \text { and in the second } \\
\qquad \mu_{\alpha} \rightarrow \beta_{\beta \gamma \delta} \text { and } \mu_{\beta} \rightarrow \mu_{\alpha}\end{array}$ & {$[\mu \beta]^{1,1}$} \\
\hline$\left[\mu^{2}\right]^{0,2}$ & 1 & $P_{\alpha \beta} \rightarrow P_{\alpha \beta \gamma}$ & $\mu_{\beta} \rightarrow \alpha_{\beta \gamma}$ & {$[\mu \alpha]^{0,2}$} \\
\hline$\left[\mu^{2}\right]^{0,2}$ & $\frac{1}{4}$ & $P_{\alpha \beta} \rightarrow P_{\alpha \beta \gamma \delta}$ & $\begin{array}{c}\mu_{\alpha} \rightarrow \alpha_{\alpha \beta}, \mu_{\beta} \rightarrow \alpha_{\gamma \delta} \\
\lambda_{a b}^{ \pm \sigma} \rightarrow \lambda_{a b}^{ \pm 23}, \lambda_{b}^{ \pm \sigma} \rightarrow \lambda_{b}^{ \pm 23}, \lambda_{c}^{ \pm \sigma} \rightarrow \lambda_{c}^{ \pm 23} \\
\lambda_{\alpha}^{ \pm \sigma} \rightarrow \lambda_{\alpha}^{ \pm 23}\end{array}$ & {$\left[\alpha^{2}\right]^{0,2}$} \\
\hline$\left[\mu^{2}\right]^{0,2}$ & $\frac{1}{3}$ & $P_{\alpha \beta} \rightarrow P_{\alpha \beta \gamma \delta}$ & $\mu_{\beta} \rightarrow \beta_{\beta \gamma \delta}$ & {$[\mu \beta]^{0,2}$} \\
\hline
\end{tabular}

expression for $\left[\mu^{4}\right]^{0,2}$. To be more precise, with the abbreviations in the footnote to Table I and changing indices where appropriate, the essential form of $\left[\mu^{4}\right]^{2,0}$ is

$$
\begin{gathered}
\left(\partial \mu_{\alpha} / \partial Q_{a}\right)\left(\partial^{2} \mu_{\beta} / \partial Q_{a} \partial Q_{c}\right)\left(\partial^{2} \mu_{\gamma} / \partial Q_{c} \partial Q_{d}\right) \\
\times\left(\partial \mu_{\delta} / \partial Q_{d}\right) \lambda_{a}^{ \pm} \lambda_{c}^{ \pm 23} \lambda_{d}^{ \pm 3}
\end{gathered}
$$

whereas for $\left[\mu^{4}\right]^{1,1}$ it is

$$
\begin{gathered}
F_{a b c}\left(\partial \mu_{\alpha} / \partial Q_{a}\right)\left(\partial \mu_{\beta} / \partial Q_{b}\right)\left(\partial^{2} \mu_{\gamma} / \partial Q_{c} \partial Q_{d}\right) \\
\times\left(\partial \mu_{\delta} / \partial Q_{d}\right) \lambda_{a}^{ \pm \sigma} \lambda_{b}^{ \pm 1} \lambda_{c}^{ \pm 23} \lambda_{d}^{ \pm 3} .
\end{gathered}
$$

It is apparent that increasing the mechanical anharmonicity and decreasing the electrical anharmonicity simultaneously by one order introduces $\lambda_{b}^{ \pm 1}$; changes the second derivative of $\mu_{\beta}$ (associated with $\omega_{1}$ ) with respect to $Q_{a}$ and $Q_{c}$ to a single derivative with respect to $Q_{b}$ (note the recurrence of $b$ ); and introduces the cubic force constant $F_{a b c}$. Repeating this step in a consistent way we have $\partial Q_{c} \partial Q_{d} \rightarrow \partial Q_{e}$ and, then, introducing $\lambda_{e}^{ \pm 2}$ and $F_{c d e}$ gives the essential form of $\left[\mu^{4}\right]^{0,2}$ as

$$
\begin{gathered}
F_{c d e} F_{a b c}\left(\partial \mu_{\alpha} / \partial Q_{a}\right)\left(\partial \mu_{\beta} / \partial Q_{b}\right)\left(\partial \mu_{\gamma} / \partial Q_{e}\right) \\
\times\left(\partial \mu_{\delta} / \partial Q_{d}\right) \lambda_{a}^{ \pm \sigma} \lambda_{b}^{ \pm 1} \lambda_{c}^{ \pm 23} \lambda_{d}^{ \pm 3} \lambda_{e}^{ \pm 2} .
\end{gathered}
$$

With the known numerical factor for the static quantity and with some rearrangement of the indices, the formula for the " $F^{2}$,' part of $\left[\mu^{4}\right]^{0,2}$ given in Table I was obtained.
In deriving the general expressions it has been very helpful to compare with the static limit. ${ }^{8-10}$ This led us to discover that the diatomic formulas of Ref. 9 are only approximate because the anharmonic terms in Eq. (5) of that reference were not carried through in deriving Eqs. (11b) and (11c). These terms were included subsequently in the corresponding treatment of polyatomics. ${ }^{10}$ However, in the latter case the fact that the field-dependent harmonic force constant matrix contains off-diagonal elements, when expressed in terms of field-free normal coordinates, was ignored. One can determine the effect of these off-diagonal matrix elements on the field-dependent harmonic vibrational frequencies, which are the quantities of interest, by carrying out an appropriate perturbation treatment. Thus, we write

$$
\mathbf{T}^{\dagger}(\mathbf{F}) \mathbf{K}(\mathbf{F}) \mathbf{T}(\mathbf{F})=\omega^{2}(\mathbf{F}),
$$

where $\mathbf{K}(\mathbf{F})$ is the field-dependent harmonic force constant matrix, $\omega(\mathbf{F})$ is the diagonal matrix of field-dependent harmonic frequencies, and $\mathbf{T}(\mathbf{F})$ is a unitary transformation,

$$
\mathbf{T}^{\dagger}(\mathbf{F}) \mathbf{T}(\mathbf{F})=\mathbf{1}
$$

All quantities are, then, expanded as a power series in $\mathbf{F}$ with $\mathbf{K}(0)$ being diagonal and $\mathbf{T}(0)=\mathbf{1}$. After some simplification it can be shown that the resulting formulas agree exactly with those given in Tables I and II when the static limit is taken (see the Appendix for an example). Thus, the equiva- 
lence between the perturbation theory ${ }^{6}$ and finite field ${ }^{10}$ approaches to static vibrational hyperpolarizabilities demonstrated in Ref. 8 is now extended to polyatomic molecules. A similar extension can be made for the infinite frequency limit.

To conclude, we have presented general (dynamic) expressions, complete through second order in perturbation theory, for the vibrational polarizability $\left(\alpha^{v}\right)$, and the first and second vibrational hyperpolarizability $\left(\beta^{v}\right.$ and $\left.\gamma^{v}\right)$. The only additional contributions from the effects of vibration to this order arise from zero-point vibrational averaging.

\section{ACKNOWLEDGMENTS}

D.M.B. acknowledges financial support from the Natural Sciences and Engineering Research Council of Canada.
J.M.L. was supported by the Generalitat de Catalunya through the CIRIT Project No. FI/95-5101.

\section{APPENDIX}

If the vibrational potential is expanded in terms of fieldfree normal coordinates, $\mathbf{Q}$, then the off-diagonal quadratic force constants will no longer vanish when an external field is present. The effect of these force constants on the harmonic vibrational frequencies and, hence, on the linear and nonlinear vibrational polarizabilities, can be evaluated through a perturbation treatment of Eqs. (10) and (11). The purpose of this Appendix is to illustrate how this can be accomplished using the linear polarizability as an example.

Following the procedure of Ref. 10, the field-dependent quadratic force constant matrix for small vibrations about the field-dependent equilibrium geometry, $\mathbf{R}_{F}$, may be written as

$$
\begin{aligned}
K_{i j}(\mathbf{F})= & 2 a_{20}^{i i} \delta_{i j}+\sum_{\alpha}^{x, y, z}\left[2 a_{21}^{i j, \alpha}-6 \sum_{k=1}^{3 N-6} a_{30}^{i j k} q_{1}^{k, \alpha}\right] F_{\alpha}+\sum_{\alpha, \beta}^{x, y, z}\left[2 a_{22}^{i j, \alpha \beta}-6 \sum_{k=1}^{3 N-6} a_{30}^{i j k} q_{2}^{k, \alpha \beta}-6 \sum_{k=1}^{3 N-6} a_{31}^{i j k, \alpha} q_{1}^{k, \beta}\right. \\
& \left.+12 \sum_{k, l=1}^{3 N-6} a_{40}^{i j k l} q_{1}^{k, \alpha} q_{1}^{l, \beta}+6 \sum_{k, l=1}^{3 N-6} a_{30}^{i j k} a_{21}^{k l, \alpha} q_{1}^{l, \beta}\left(a_{20}^{k k}\right)^{-1}-9 \sum_{k, l, m=1}^{3 N-6} a_{30}^{i j k} a_{30}^{k l m} q_{1}^{l, \alpha} q_{1}^{m, \beta}\left(a_{20}^{k k}\right)^{-1}\right] F_{\alpha} F_{\beta}+\cdots,
\end{aligned}
$$

where

$$
\begin{aligned}
& a_{n m}^{i_{1} \cdots i_{n}, j_{1} \cdots j_{m}} \\
& \quad=\frac{1}{n ! m !}\left(\frac{\partial^{(n+m)} V\left(Q_{1}, \ldots, Q_{3 N-6}, F_{x}, F_{y}, F_{z}\right)}{\partial Q_{i_{1}} \cdots \partial Q_{i_{n}} \partial F_{j_{1}} \cdots \partial F_{j_{m}}}\right)_{\mathbf{Q}=\mathbf{0 , F}=\mathbf{0}},
\end{aligned}
$$

$q_{1}^{\lambda, \alpha}=a_{11}^{\lambda, \alpha} / 2 a_{20}^{\lambda \lambda} \quad$ and $\quad q_{2}^{\lambda, \alpha \beta}=a_{12}^{\lambda, \alpha \beta} / 2 a_{20}^{\lambda \lambda}$.

The term linear in $\mathbf{F}$ is considered to be first order; the term quadratic in $\mathbf{F}$ is second order; and so forth. Through second order the harmonic vibrational frequencies from Eq. (13) are given by,

$$
\omega_{i}^{2}(F) \cong \omega_{i}^{2}(0)+\sum_{\alpha}\left[\omega_{i}^{2}\right]^{\alpha} F_{\alpha}+\sum_{\alpha, \beta}\left[\omega_{i}^{2}\right]^{\alpha \beta} F_{\alpha} F_{\beta},
$$

with

$$
\omega_{i}^{2}(0)=K_{i i}(0)=2 a_{20}^{i i},
$$

$$
\left[\omega_{i}^{2}\right]^{\alpha}=K_{i i}^{\alpha}=2 a_{21}^{i i, \alpha}-6 \sum_{k=1}^{3 N-6} a_{30}^{i i k} q_{1}^{k, \alpha},
$$

and

$$
\begin{aligned}
{\left[\omega_{i}^{2}\right]^{\alpha \beta}=} & K_{i i}^{\alpha \beta}+\left[\mathbf{T}^{\alpha^{\dagger}} \mathbf{K}^{\beta}\right]_{i i}+\left[\mathbf{K}^{\alpha} \mathbf{T}^{\beta}\right]_{i i}+\left[\mathbf{T}^{\alpha^{\dagger}} \mathbf{K}(0) \mathbf{T}^{\beta}\right]_{i i} \\
& +2 T_{i i}^{\alpha \beta} K_{i i}(0)
\end{aligned}
$$

In deriving Eqs. (A4)-(A6) we have used the fact that $\mathbf{K}$ is Hermitian; $\mathbf{K}(0)$ is diagonal; and $\mathbf{T}(\mathbf{F})=\mathbf{1}+\sum \mathbf{T}^{\alpha} F_{\alpha}$ $+\Sigma T^{\alpha \beta} \mathbf{F}_{\alpha} F_{\beta}+\cdots$ is unitary. Thus,

$$
T_{i i}^{\alpha}=0, \quad T_{i j}^{\alpha}=K_{i j}^{\alpha}\left[K_{j j}(0)-K_{i i}(0)\right]^{-1} \quad(j \neq i)
$$

and

$$
T_{i i}^{\alpha \beta}=-\frac{1}{2} \sum_{j \neq i} T_{j i}^{\alpha} T_{j i}^{\beta} .
$$

From Eqs. (A6) - (A8) one obtains

$$
\begin{aligned}
{\left[\omega_{i}^{2}\right]^{\alpha \beta}=} & 2 a_{22}^{i i, \alpha \beta}-6 \sum_{j=1}^{3 N-6} a_{30}^{i i j} q_{2}^{j, \alpha \beta}-6 \sum_{j=1}^{3 N-6} a_{31}^{i i j, \alpha} q_{1}^{j, \beta}+12 \sum_{j, k=1}^{3 N-6} a_{40}^{i i j k} q_{1}^{j, \alpha} q_{1}^{k, \beta}+6 \sum_{j, k=1}^{3 N-6} a_{30}^{i i j} a_{21}^{j k, \alpha} q_{1}^{k, \beta}\left(a_{20}^{j j}\right)^{-1} \\
& -9 \sum_{j, k, l=1}^{3 N-6} a_{30}^{i i j} a_{30}^{j k l} q_{1}^{k, \alpha} q_{1}^{l, \beta}\left(a_{20}^{j j}\right)^{-1}-4 \sum_{j \neq i=1}^{3 N-6} a_{21}^{i j, \alpha} a_{21}^{i j, \beta}\left(A_{20}^{i j}\right)^{-1}+24 \sum_{j \neq i, k=1}^{3 N-6} a_{21}^{i j, \alpha} a_{30}^{i j k} q_{1}^{k, \beta}\left(A_{20}^{i j}\right)^{-1} \\
& -36 \sum_{j \neq i, k, l=1}^{3 N-6} a_{30}^{i j k} a_{30}^{i j l} q_{1}^{k, \alpha} q_{1}^{k, \beta}\left(A_{20}^{i j}\right)^{-1}
\end{aligned}
$$

where $A_{20}^{i j}=2\left(\sqrt{a_{20}^{i}}+\sqrt{a_{20}^{j}}\right)^{2}$. 
The second-order correction to the static linear polarizability [i.e., $\left[\mu^{2}\right]^{\mathrm{II}}$ in Eq. (7) of the main text with $\left.\omega_{\sigma}=\omega_{1}=0\right]$ may be found ${ }^{8,10}$ by taking the second derivative of the harmonic vibrational energy,

$$
\frac{1}{2} \sum_{i}\left[\frac{\partial^{2} \omega_{i}(\mathbf{F})}{\partial F_{\alpha} \partial F_{\beta}}\right]_{\mathbf{F}=\mathbf{0}}=\frac{1}{4} \sum_{i}^{3 N-6} \frac{1}{\omega_{i}(0)}\left[\left(\frac{\partial^{2} \omega_{i}^{2}(\mathbf{F})}{\partial F_{\alpha} \partial F_{\beta}}\right)_{\mathbf{F}=\mathbf{0}}-\frac{1}{2 \omega_{i}^{2}(0)}\left(\frac{\partial \omega_{i}^{2}(\mathbf{F})}{\partial F_{\alpha}}\right)_{\mathbf{F}=\mathbf{0}}\left(\frac{\partial \omega_{i}^{2}(\mathbf{F})}{\partial F_{\beta}}\right)_{\mathbf{F}=\mathbf{0}}\right]
$$

which (after changing sign) is equal to $\Delta \alpha^{\mathrm{ZPVA}}+\left[\mu^{2}\right]^{\mathrm{II}}$. Using $\omega_{i}^{2}(\mathbf{F})$ from Eqs. (A4), (A5), and (A9) in Eq. (A10) and

$$
\Delta \alpha_{\alpha \beta}^{\mathrm{ZPVA}}=-\sum_{i=1}^{3 N-6}\left(2 a_{20}^{i i}\right)^{-1 / 2}\left[a_{22}^{i i, \alpha \beta}-3 \sum_{j=1}^{3 N-6} a_{30}^{i i j} q_{2}^{j, \alpha \beta}\right],
$$

the result is

$$
\begin{aligned}
{\left[\mu^{2}\right]^{\mathrm{II}}=} & \frac{1}{4} \sum P_{\alpha \beta} \sum_{i=1}^{3 N-6}\left(2 a_{20}^{i i}\right)^{-1 / 2}\left[6 \sum_{j=1}^{3 N-6} a_{31}^{i i j, \alpha} q_{1}^{j, \beta}-12 \sum_{j, k=1}^{3 N-6} a_{40}^{i i j k} q_{1}^{j, \alpha} q_{1}^{k, \beta}-6 \sum_{j, k=1}^{3 N-6} a_{30}^{i i j} a_{21}^{j k, \alpha} q_{1}^{k, \beta}\left(a_{20}^{j j}\right)^{-1}\right. \\
& +9 \sum_{j, k, l=1}^{3 N-6} a_{30}^{i j j} a_{30}^{j k l} q_{1}^{k, \alpha} q_{1}^{l, \beta}\left(a_{20}^{j j}\right)^{-1}+4 \sum_{j=1}^{3 N-6} a_{21}^{i j, \alpha} a_{21}^{i j, \beta}\left(A_{20}^{i j}\right)^{-1}-24 \sum_{j, k=1}^{3 N-6} a_{21}^{i j, \alpha} a_{30}^{i j k} q_{1}^{k, \beta}\left(A_{20}^{i j}\right)^{-1} \\
& \left.+36 \sum_{j, k, l=1}^{3 N-6} a_{30}^{i j k} a_{30}^{i j l} q_{1}^{k, \alpha} q_{1}^{l, \beta}\left(A_{20}^{i j}\right)^{-1}\right]
\end{aligned}
$$

A comparison with the sum $\left[\mu^{2}\right]^{2,0}+\left[\mu^{2}\right]^{1,1}+\left[\mu^{2}\right]^{0,2}$ taken from Table I reveals that the two expressions are identical.

${ }^{1}$ D. M. Bishop, Adv. Chem. Phys. (to be published).

${ }^{2}$ See Ref. 1 and B. Kirtman, J. M. Luis, and D. M. Bishop, J. Chem. Phys. 108, 10008 (1998), preceding paper.

${ }^{3}$ D. S. Elliott and J. F. Ward, Mol. Phys. 51, 45 (1984).

${ }^{4}$ D. M. Bishop, M. Hasan, and B. Kirtman, J. Chem. Phys. 103, 4157 (1995).
${ }^{5}$ D. M. Bishop and E. K. Dalskov, J. Chem. Phys. 104, 1004 (1996).

${ }^{6}$ D. M. Bishop and B. Kirtman, J. Chem. Phys. 95, 2646 (1991); 97, 5255 (1992).

${ }^{7}$ D. M. Bishop, B. Kirtman, and B. Champagne, J. Chem. Phys. 107, 1501 (1997).

${ }^{8}$ J. Martí and D. M. Bishop, J. Chem. Phys. 99, 3860 (1993).

${ }^{9}$ J. M. Luis, J. Martí, M. Duran, and J. L. Andrés, J. Chem. Phys. 102, 7573 (1995).

${ }^{10}$ J. M. Luis, M. Duran, and J. L. Andrés, J. Chem. Phys. 107, 1501 (1997). 